\title{
Hydrogen as an energy carrier: prospects and challenges
}

\begin{abstract}
This paper provides an insight to the feasibility of adopting hydrogen as a key energy carrier and fuel source in the near future. It is shown that hydrogen has several advantages, as well as few drawbacks in using for the above purposes. The research shows that hydrogen will be a key player in storing energy that is wasted at generation stage in large-scale power grids by off-peak diversion to dummy loads. The estimations show that by the year of 2050 there will be a hydrogen demand of over 42 million metric tons or 45 billion gallon gasoline equivalent (GGE) in the United States of America alone which can fuel up 342 million light-duty vehicles for $51 \times 1011$ miles $(82 \times 1011 \mathrm{~km})$ travel per year. The production at distributed level has also been discussed. The paper also presents the levels of risk in production, storage and distribution stages and proposes possible techniques to address safety issues. It is shown that the storage in small to medium scale containers is much economical compared to doing the same at large-scale containers. The study concludes that hydrogen has a promising future to be a highly feasible energy carrier and energy source itself at consumer level.
\end{abstract}

Keyword: Renewable energy; Hydrogen; Electrolysis; Energy conversion; Fuel 